\title{
La Investigación-Acción-Participante como Estrategia para la Capacitación y Evaluación en Educación Sanitaria
}

\author{
Action-Oriented Participant Research as a Strategy for Training and \\ Evaluation in Health Education
}

\begin{abstract}
Gloria Perdomo ${ }^{1}$
PERDOMO, G. Action-Oriented Participant Research as a Strategy for Training and Evaluation in Health Education. Cad. Saúde Públ., Rio de Faneiro, 10 (3): 331-338, Ful/Sep, 1994.

In this article we present the results of a study on educational practices at the "Escuela de Malariología y Saneamiento Ambiental `Dr. Arnoldo Gabaldón”" (EMSA), a pioneering institution in training health personnel, pertaining to the Ministry of Health and Social Assistance in Venezuela.

This study was developed as an experiment in action-oriented participant research, i.e. authorities, teachers, and students were committed to an evaluation of the educational practices in which they were involved. The main results of this cooperative inquiry were: a theoretical reconstruction of the models of health education employed by EMSA; a critical analysis of those models; and the design and testing of an alternative model centered on community participation.
\end{abstract}

Key words: Health Education; Community Participation; Action-Oriented Research; Education for Participation; Educational Models

\section{INTRODUCCION}

La importancia de la Educación Sanitaria para los programas de protección, fomento o recuperación de la salud, es una afirmación indiscutible en los ambientes académicos, científicos, institucionales y comunitarios. Pero a pesar de su reconocida importancia, esta Educación casi siempre se desarrolla como una práctica eminentemente empírica, cuyas estrategias y acciones suelen orientarse por la rutina, la improvisación o la adaptación acrítica de modelos foráneos.

La investigación que aquí presentamos quiso hacer un aporte a la reflexión crítica acerca de las propuestas de Educación para la Salud, que no sólo resultan relevantes en el ámbito de la formación del personal sanitario, sino que pueden ilustrar y

${ }^{1}$ Lab. de Investigaciones Sociales de la Universidad Central de Venezuela. Apartado 47795, Caracas, 1040-A Venezuela. esclarecer las principales dificultades que confrontan las distintas experiencias de educación de adultos (en especial, aquéllas que se orientan a promover la capacitación, organización y participación de las comunidades).

Con esta perspectiva, diseñamos una propuesta de investigación-acción-participante, concertada con la Escuela de Malariología y Saneamiento Ambiental "Dr. Arnoldo Gabaldón" (EMSA), institución creada desde 1936 para la formación de personal especializado en las labores de salud pública, y que está adscrita a la Dirección General Sectorial del Subsistema Sanitario Ambiental del Ministerio de Sanidad y Asistencia Social en Venezuela (DGSSSA).

Más específicamente, el estudio integró la reflexión crítica y la acción coooperativa de directivos, docentes y alumnos en una experiencia de investigación participativa dirigida a descubrir, conceptualizar y analizar críticamente, las formulaciones 
teóricas implícitas en las experiencias educativas que se realizan en la EMSA.

El esfuerzo investigativo se orientó prioritariamente al logro de los siguientes objetivos:

- Describir y analizar los modelos educativos implícitos en las experiencias de educación sanitaria promovidas en la EMSA en su curso de asistentes en educación sanitaria (AES) y en sus programas educativos dirigidos a las comunidades.

- Evaluar esos modelos educativos en términos de su adecuación a su entorno ecológico, de la coherencia entre su práctica y sus propuestas normativas, y de su potencialidad o efectividad para promover la participación comunitaria en la preservación y la vigilancia de la salud.

- Proponer estrategias educativas que potencien los recursos y capacidades comunitarios $\mathrm{y} / \mathrm{o}$ familiares para la prevención de las enfermedades tropicales.

\section{METODOS EMPLEADOS}

Al plantear esta investigación, nos situamos en el amplio contexto epistemológico que ofrece la sociología cualitativa. Más específicamente, optamos por el desarrollo de teorías sustantivas (Glaser \& Strauss, 1967), al estudiar sistemáticamente ciertas prácticas de educación sanitaria, procurando derivar de ellas las definiciones, supuestos y premisas que las expresan, sin la mediación de hipótesis o esquemas interpretativos previos, que pudiesen distorsionar o encasillar el estudio a realizar.

Para estos fines, la investigaciónacción-participativa resultó ser el recurso metodológico más idóneo, porque integró el aporte crítico de los distintos participantes en las experiencias analizadas, asegurando así la validez ecológica y la pertinencia social de los resultados obtenidos.

Nuestra propuesta de investigación cumplió con el desarrollo de las siguientes fases de trabajo:
1) Fase Inicial o Exploratoria, en la cual se utilizaron las técnicas de la observación participante, las entrevistas no estructuradas y la investigación documental para lograr las siguientes metas:

- La familiarización del investigador con la realidad social e institucional que caracteriza a la Escuela de Malariología y Saneamiento Ambiental "Dr. Arnoldo Gabaldón", lo que equivale a la propuesta metodológica del "estudio preliminar y provisional" en el enfoque psicosocial de Guy Le Boterf (1981), o a la "negociación del acceso" en la etnografía educativa expuesta por Woods (1987).

- El estudio de los antecedentes históricos y teóricos, a través de la investigación documental y de entrevistas focalizadas a sanitaristas, autoridades y expertos en materia de educación sanitaria.

- el análisis del proyecto inicial de esta investigación por parte del personal directivo y docente de la EMSA, a los fines de verificar su adecuación a las necesidades y prioridades de la institución y también para acordar estrategias que permitieran la incorporación de docentes, directivos y alumnos en las distintas etapas del estudio a realizar. Para ello fué preciso la realización de entrevistas individuales y de dos reuniones de trabajo en las cuales se buscaba integrar un equipo dispuesto a participar activamente en esta experiencia de investigación cooperativa.

\section{2) Fase Diagnóstica o Descriptiva, que} permitió documentar y analizar las prácticas educativas que adelanta la EMSA en su Curso de Asistentes en Educación Sanitaria. Para ello se organizó un complejo proceso de recolección de datos que tenía el doble propósito de lograr una descripción preliminar de la propuesta educativa AES-EMSA y de iniciar su crítica a partir de su contrastación con las características y demandas de los contextos (académico, ocupacional, social y comunitario) a los cuales debe adecuarse la formación 
profesional. Así, el proceso de recolección de datos abarcó las siguientes actividades:

\section{a) El estudio de los modelos educativos}

AES-EMSA, a través de reuniones, talleres de reflexión y de la observación participante de las actividades administrativas, académicas y comunitarias que se realizaron en el XVIII Curso AES-EMSA. Pero también fué necesario el análisis de contenido de los programas y materiales que orientan el trabajo de la EMSA en Educación Sanitaria, y la realización de entrevistas individuales y colectivas con docentes, alumnos, autoridades y exdirectivos de la institución.

b) El estudio del perfil académico del educador sanitario, para lo cual se realizaron entrevistas a funcionarios responsables de las instituciones de planificación de la educación venezolana, y se hizo un análisis documental de la oferta académica vigente en el país para la formación en el campo de la salud.

c) La realización de una encuesta ocupacional, aplicada en 23 instituciones de atención y educación sanitaria existentes en Venezuela, para conocer las expectativas y demandas del mercado de trabajo en relación a la formación de un especialista en educación para la salud.

d) Una investigación documental sobre los materiales publicados por expertos y por organismos científicos en el area de la educación sanitaria, para conocer el perfil del educador sanitario sugerido por ellos.

e) Un análisis de la realidad social y comunitaria a la cual debe adecuarse la formación profesional que realiza la EMSA, que se cumplió inicialmente, con el proceso de evaluación entre comunidades pertenecientes a los estados Carabobo y Aragua, para la selección de las poblaciones-sedes del trabajo de campo que realizarían los participantes del XVIII Curso AES-EMSA. Posteriormente, fué posible un conocimiento más detallado de la realidad comunitaria, con el desarrollo de los planes de investigación adelantados por los distintos equipos de pasantía.

3) Fase de Autorreflexión Y Acción, orientada a profundizar el conocimiento descriptivo obtenido con el desarrollo de la fase anterior. Así, en esta fase privilegiamos las estrategias de investigación-acción (De Schutter et al., 1981; Mosser, 1978) y de evaluación participativa (Guba \& Lincoln, 1990), para desarrollar distintos planes de trabajo que integraron el conocimiento crítico y la acción reformadora de docentes, alumnos y miembros de las comunidades.

Concretamente, el estudio se focalizó en el análisis de los siguientes "aspectos problemáticos" de la práctica educativa que realiza el curso AES-EMSA:

- El proceso de admisión.

- La asignatura "Naturaleza del proceso Educativo".

- La metodología de trabajo comunitario que orienta el trabajo de campo que se realiza en las pasantías.

- Los procedimientos de evaluación y de exposición de estas pasantías.

Es importante destacar que esta fase de investigación surge de la revisión del proyecto inicial, para adecuarlo a las necesidades y prioridades de los docentes y alumnos que se integraban al proceso investigativo. El estudio se transformó así en un esfuerzo cooperativo dirigido a buscar alternativas frente a los problemas de la práctica educativa, a incrementar y profundizar la explicación de esta realidad y a capacitar a los participantes en estrategias de investigación-acción.

\section{4) Fase de Evaluación y Sistematización} que permitió la elaboración teórica de los modelos de educación sanitaria identificados, y su contrastación con las propuestas de "la educación como práctica de concientización" (Freire, 1975) y de "la ciencia educativa crítica” expuesta por Carr \& Kemmis (1988). Ello implicó un esfuerzo importante para la ordenación de distintos datos e interpretaciones y para su integración final en la perspectiva histórica y conceptual que aquí reportamos.

Sin embargo, el carácter democrático y cooperativo del proyecto de investigación propuesto requirió de un esfuerzo adicional para la crítica y confrontación de sus 
resultados parciales y finales con los distintos protagonistas de las prácticas educativas analizadas. Con estos propósitos, en distintos momentos del estudio se realizaron varias reuniones para el análisis de los datos obtenidos por el equipo investigador, también se efectuaron asambleas en las que el trabajo de pasantías fué evaluado por las comunidades. Cuatro meses después del curso AES se realizó un taller con los egresados del curso para conocer el impacto de la experiencia realizada y, con posterioridad, el informe final de la investigación fué presentado y discutido por los directivos y educadores sanitarios de la EMSA y de la Dirección General Sectorial de Malariología y Saneamiento Ambiental del MSAS.

Durante estas cuatro fases de trabajo se hizo un registro de cada una de las actividades utilizando para ello los diarios de campo, las actas de reuniones, las grabaciones, las crónicas, y en menor medida, las listas de cotejo, las fotografías y las guías de entrevista.

Los datos así colectados fueron validados a través del uso de algunas de las siguientes estrategias de "triangulación” (Woods, 1987):

a. La búsqueda y confrontación de las informaciones y opiniones que aportan diferentes participantes en las experiencias que se estudian.

b. El uso simultáneo de varias estrategias de investigación, las cuales deberían proporcionar información complementaria o coincidente (Glaser \& Strauss, 1967) en relación a los problemas investigados.

Para la revisión inicial de los instrumentos estructurados de investigación (sondeo de opinión, la encuesta ocupacional y la escala de evaluación) recurrimos al juicio de expertos y a una prueba piloto para evaluar la versión corregida de cada uno de ellos.

\section{RESULTADOS}

1) El estudio realizado demuestra que no existe una única concepción educativa que explique u oriente las prácticas formativas adelantadas por la EMSA. Los datos obtenidos permiten describir tres modelos de educación sanitaria que se desarrollan en este contexto institucional con distintos planteamientos conceptuales y metodológicos tanto en Educación para la Salud como en lo referido a la formación del personal especializado en ese campo.

2) Esas tres concepciones educativas surgen y se desarrollan en contextos sociales claramente diferenciados en la realidad venezolana (tomando en cuenta factores socioeconómicos, políticos y epidemiológicos); sin embargo, no se han sucedido cronológicamente, sino que coexisten, se superponen y coliden en las experiencias educativas que realizan los programas y servicios adscritos a la DGSSSA del MSAS.

3) Los "modelos de educación sanitaria" identificados son los siguientes:

\section{Educación como Atención Sanitaria}

Este enfoque ha sido la práctica educativa de mayor arraigo y generalización en los distintos servicios de la DGSSSA. Desde su óptica, la educación constituye un tratamiento aplicable a poblaciones reacias a los programas, para motivar en ellas los hábitos de vida higiénica. El educador actúa como agente de salud dispuesto a enseñar (mostrar, decir, transmitir) y masificar el conocimiento médico-sanitario mientras la institución trabaja en la comunidad instalando un servicio o realizando campañas u operativos para preservar o recuperar la salud de los pobladores. Generalmente, el rol de la comunidad en este modelo se limita a la solicitud o recepción de los servicios educativos o asistenciales que le dispensa la institución.

\section{Educación para el Desarrollo Comunal Con una clara influencia del conductismo y del enfoque biomédico de los problemas de salud, este segundo modelo define la educación sanitaria como una técnica dirigida a programar y controlar cambios de actitudes, costumbres y comportamientos de la población con respecto a su salud. Desde esta}


óptica, al educador sanitario le corresponde actuar como tecnólogo social, preparado para modelar o corregir las actitudes y comportamientos de individuos o comunidades que se diagnostican como enfermos o ignorantes y quienes sólo pueden sanar si participan en los programas institucionales. La consecuencia práctica de este enfoque educativo es el fracaso de programas que son percibidos como impuestos, extraños a la realidad local, pero sobre todo, ajenos a la población, que participa sólo mientras está presente el agente de cambio con sus reforzadores.

\section{Educación para la Autogestión Comunitaria en Salud}

Con la influencia de los principios formulados por la Organización Mundial de la Salud (OMS) en su Conferencia Internacional de Alma Ata (OMS, 1978), y de la educación popular expuesta por Paulo Freire (1975a, 1984), se inicia en la EMSA la crítica de los estilos tecnocráticos e impositivos en las prácticas de educación sanitaria. Así, en los últimos años se trabaja por la definición conceptual y metodológica de un nuevo enfoque educativo cuyos objetivos proritarios son los de fortalecer y desarrollar las capacidades de la población para la acción crítica, concertada y cooperativa frente a los problemas de salud de sus comunidades. Para ampliar la información relativa a estos tres modelos, en la página siguiente presentamos un cuadro que resume algunos de los principales elementos que los caracterizan (Cuadro 1).

4) El estudio crítico demostró el carácter pragmático e inmediatista de las formulaciones planteadas en el primer modelo, y sus efectos perversos al promover una conciencia sanitaria que concentra todo el poder y la responsabilidad por los problemas de salud en el personal de la institución sanitaria. En el caso del segundo modelo, se destacó lo inadecuado de su planteamiento tecnocrático, para responder a las exigencias de la educación no-formal y a las características propias de las realidades comunitarias. Al examinar la propuesta de autogestión comunitaria en salud, se concluyó afirmando que la sólidez teórica de su enfoque educativo no tiene correspondencia con una propuesta metodológica que la desarrolle o exprese en las prácticas formativas que realiza la EMSA.

5) Con el análisis teórico se reconocieron las coincidencias entre los dos modelos de "Educación para la atención sanitaria" y "Educación para el Desarrollo Comunal" con la "concepción bancaria de la educación" (Freire, 1975), mientras que el tercer modelo declara explícitamente su adscripción a las formulaciones normativas propuestas por este autor en su concepción dialógica de la enseñanza. Sin embargo, para los tres modelos descritos sería válida la crítica que este autor brasilero hace a sus primeros trabajos, por la ausencia de un análisis político y social que sitúe históricamente cada propuesta educativa (Torres, 1986) y la advertencia señalada por Carr \& Kemmis (1988), en relación a que sólo en condiciones sociales e institucionales adecuadas es posible la autorreflexión crítica y la acción reformadora que permita democratizar y reformar las experiencias educativas.

6) Al analizar la Investigación-AcciónParticipante como recurso teóricometodológico para la formación del trabajador de salud pública, se concluye que sus premisas teóricas resultan consistentes con la concepción educativa a la que se adscribe el tercer modelo de autogestión comunitaria en salud.

La prueba práctica de un diseño cooperativo de investigación-acciónparticipante demostró su pertinencia para la formación de un educador curioso, crítico y dispuesto al diálogo con la población a la que sirve. Pero ante todo, resaltó su adecuación y factibilidad para favorecer el desarrollo de distintos niveles de participación comunitaria en las actividades (culturales, organizativas, sociosanitarias) que se realizan en las pasantías del curso AES-EMSA, a partir de un proyecto concertado entre los grupos comunitarios, los pasantes y algunos funcionarios de las instituciones locales. 


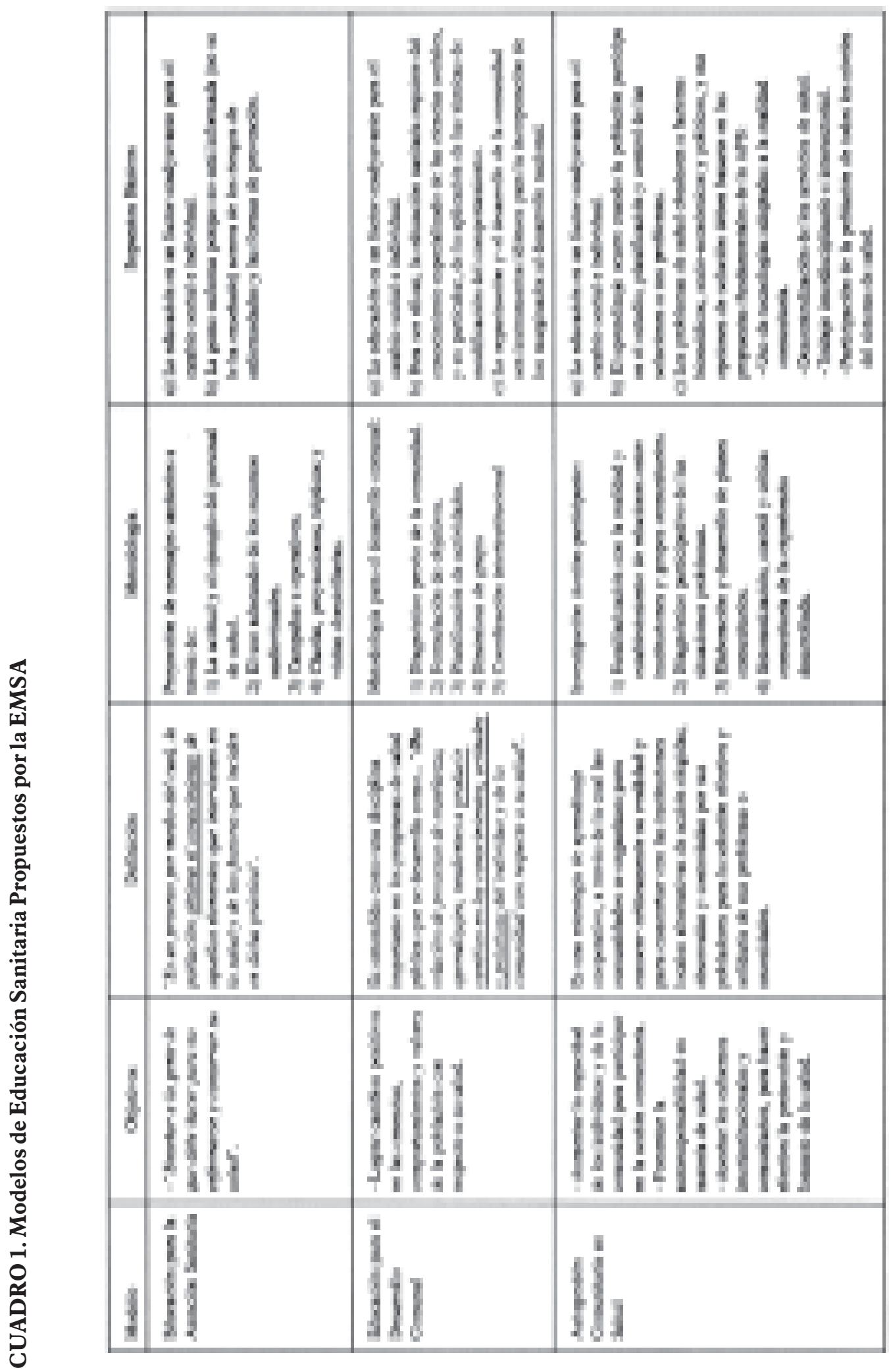


7) El desarrollo de cualquier propuesta en Educación para la Salud requiere la presencia de condiciones mínimas que aseguren su válidez y pertinencia social. En el caso de la DGSSSA, ello significa cubrir tres exigencias básicas:

a. acreditar la formación académica del educador sanitario, para lo cual es necesario el rediseño curricular del Curso AES-EMSA, y emprender un proyecto institucional dirigido a crear una carrera universitaria en este campo;

b. crear un espacio laboral propio para el trabajo educativo, lo que implica asignarle un lugar en la jerarquía organizacional, ocupar a un personal a dedicación exclusiva, implementar programas formativos sustentados con los recursos necesarios (cargos, equipos, presupuestos) y desarrollar actividades para la actualización profesional y para la evaluación permanente del trabajo operativo;

c. organizar y mantener servicios de educación sanitaria que aseguren una creciente participación y control de la población en las distintas fases de los programas dirigidos a la prevención y recuperación de su propia salud.

\section{CONCLUSIONES}

La investigación realizada demostró la adecuación de los estudios cualitativos para acceder al descubrimiento de la teoría implícita en las prácticas sociales. Así, con la observación sistemática de la experiencia directa, se esclareció que en la EMSA coexisten concepciones y prácticas educativas francamente contradictorias, que antagonizan y compiten tanto en la realidad de los servicios como en sus programas de formación de recursos humanos.

Esta primera conclusión permite reconocer que junto a los llamados "programas de educación para la salud", en las instituciones y comunidades, coexisten situaciones, relaciones y actividades con un importante potencial educativo, que deben ser identificadas y analizadas al momento de diseñar o realizar cualquier intento formativo: al educador sanitario le corresponde, en cada caso, apoyarse o competir con tradiciones, hábitos y costumbres arraigados en experiencias de socialización tanto o más efectivas que las actividades formativas, técnicamente planificadas.

Pero esta visión amplia de la educación nos lleva también a contradecir la afirmación según la cual, de los programas de educación para la salud sólo pueden resultar aprendizajes positivos. Al respecto, este estudio analiza los efectos perversos de los estilos pedagógicos cientificistas, impositivos o irrespetuosos de las realidades comunitarias, que resultan inútiles en términos del logro o permanencia del comportamiento saludable que buscan, pero que parecen eficaces en la enseñanza de la dependencia, del paternalismo y de la acción inmediatista en materia sanitaria.

Igualmente, del análisis realizado se confirma que la educación para la salud no puede ser decretada, ni transmitida en paquetes tecnológicos; por el contrario, se reconoce la complejidad de elementos y factores (interactivos, cognoscitivos, histórico- sociales, culturales) que la determinan y condicionan, por lo que se hace necesario recomendar que la investigación educativa se incorpore al trabajo cotidiano de las instituciones y organizaciones de salud, como estrategia imprescindible para la planificación, supervisión y evaluación de sus tareas educativas.

Para el caso específico de la EMSA, este estudio confirmó que desde el punto de vista teórico, la investigación-acción-participativa es una estrategia metodológica consistente con las propuestas educativas que buscan la autogestión comunitaria y la acción concertada e intersectorial en materia de salud pública. La prueba práctica del diseño de investigación propuesto evidenció su adecuación para promover en el personal de salud actitudes y comportamientos de respeto y solidaridad hacia las comunidades, favoreciendo así el encuentro dialógico y la interacción cooperativa entre pobladores y profesionales.

Otra discusión planteada en este estudio es 
la de si es preciso especializar a un profesional en el campo educativo, o si debe entenderse que todo trabajador de salud es, de hecho, un educador sanitario. Desde una perspectiva amplia, resulta obvia la segunda opción, lo que implica recomendar una mejor preparación de todo el personal de salud en los asuntos educativos. Pero si se es coherente con los objetivos de participación y democratización propuestos en Alma Ata, entonces educar deja de ser una tarea de transmisión o imposición de prédicas sanitarias, y se convierte en un esfuerzo investigativo, para la creación de conocimientos y de metodologías que fomenten el autoaprendizaje y el control de la comunidad sobre las situaciones y prácticas que afectan la salud de sus pobladores: ello exige un cambio importante en la concepción de los programas y en la capacitación del personal, lo que en el contexto de la DGSSSA sólo parece factible si se establece un espacio administrativo propio para la educación sanitaria y si los esfuerzos formativos se concentran en el entrenamiento especializado de un equipo comprometido a tiempo completo con esta opción educativa.

\section{RESUMEN}

\section{PERDOMO, G. La Investigación-Acción-} Participante como Estrategia para la Capacitación y Evaluación en Educación Sanitaria. Cad. Saúde Públ., Rio de Janeiro, 10 (3): 331-338, jul/set, 1994.

Presentamos aquí los resultados de un estudio acerca de las prácticas educativas promovidas por la Escuela de Malariología y Saneamiento Ambiental "Dr. Arnoldo Gabaldón" (EMSA), institución pionera en la formación de personal de salud y que está adscrita al Ministerio de Sanidad y Asistencia Social en Venezuela.

Dicho estudio se realizó como experiencia de investigación-acción-participativa al incorporar el punto de vista de autoridades, docentes y alumnos acerca de las prácticas educativas en la que ellos intervienen. Como resultados de este esfuerzo cooperativo, fué posible reconstruir teóricamente los modelos educativos implícitos en los programas de educación sanitaria de la EMSA, y a partir de su análisis crítico, se diseñaron y probaron alternativas metodológicas orientadas a lograr la autonomía de las comunidades en los asuntos referidos a la preservación de la salud de sus habitantes.

Palabras-Claves: Educación Sanitaria; Participación Comunitaria; InvestigaciónAcción; Educación para la Participación; Modelos Educativos

\section{REFERENCIAS BIBLIOGRÁFICAS}

CARR, W. \& KEMMIS, S., 1988. Teoría Crítica de la Enseñanza. Barcelona: Ediciones Martínez Roca.

FREIRE, P., 1975. La Educación como Práctica de la Libertad. XIV edición, Buenos Aires: Siglo XXI Argentina Editores. 1984. Viviendo y Aprendiendo. Caracas: Cooperativa Laboratorio Educativo. DE SCHUTTER, A.; LE BOTERF, G.; GIANOTTEN, V.; VIO GROSSI, F. \& WIT, T. (Eds.), 1981. Investigación Participativa y Praxis Rural. Lima: Mosca Azul Editores.

GLASER, B. \& STRAUSS, A., 1967. The Discovery of Grounded Theory. Chicago: Aldine Publishing Company.

GUBA, E. \& LINCOLN, I., 1990. Fourth Generation Evaluation. Newbury Park: Sage Publications.

MOSSER, H., 1978. La investigación acción como nuevo paradigma en las ciencias sociales. In: Crítica y Política en Ciencias Sociales (Simposio Mundial de Cartagena, orgs.), Tomo I (El Debate sobre Teoría y Práctica), pp. 117-140, Bogotá: Punta de Lanza.

OMS (Organización Mundial dela Salud), 1978. Alma Ata 1978: Atención Primaria de Salud. Informe de la Conferencia Internacional sobre Atención Primaria de Salud. Alma Ata, URSS, 6-12 de septiembre de 1978, Ginebra: OMS. (Serie "Salud para Todos", 1)

TORRES, R. M., 1988. Educación Popular. Un Encuentro con Paulo Freire. Buenos Aires: Centro Editor de América Latina.

WOODS, P., 1987. La Escuela por Dentro. Barcelona: Ediciones Paidós. 